\title{
Operations for parallel satellite support
}

\author{
Marcin Gnat ${ }^{1}$ and Peter Willburger ${ }^{2}$ \\ DLR, Oberpfaffenhofen, Germany, 82234
}

\begin{abstract}
In the early preparation phase for the upcoming robotic dual-satellite DLR mission several technical and operational challenges presented themselves. The mission itself shall produce advanced scientific findings for the on-orbit servicing missions. One of the satellites includes a sophisticated robotic arm with automated as well as manual operation modes. Very restrictive robotic payload requirements with respect to ground station visibilities as well as the quality of the data link became a main driver for the ground data system design. The real-time control and feedback of the robotic arm represents the particular challenge. Analysis of these requirements as well as technical and operational solutions will be presented, whereas some results are based on the successful ROKVISS mission. The usage of a dual-uplink antenna is discussed with regard to parallel operations of two satellites, here again, with already existing results provided by operations of the TanDEM-X mission. The design of the ground communication network as well as possible solutions allowing parallel robotic and housekeeping operations is shown. Results of this mission analysis and preparation are not only valuable for particular robotic, but for all dual-satellite, high data rate or realtime communication missions.
\end{abstract}

\section{Introduction}

$\mathrm{T}$ HE future of space exploration is becoming more and more interesting as well as challenging, as new fields of operation open up and many already known aspects become new facets. All this happens under permanent budget pressure and cuts. The job of engineers is to provide all these challenges with solutions, allowing permanent development. In this paper we focus on three aspects of space operations, trying to gather experiences and find solutions to consolidate them into one universal coherent operations concept.

The background for our first area of interest is the increasing problem of space debris. Even if all of the rules for debris avoidance [22, 23] would be enforced it would only keep present numbers from exponentially increasing. Therefore the topic of active debris removal is being discussed more intensive. Among several realistic concepts, based on current technological levels [24, 25], there are some concrete proposals using targeted robotic mission. They discuss the possibilities of catching the object (old or defective satellite) and perform deorbiting (in case of Low Earth Orbit [LEO]) $[1,9,26]$ or removal to the graveyard orbit in case of Geostationary Earth Orbit (GEO) [9, 26]. In specific cases, where a defective satellite is very valuable and there is a possibility to repair the issue, the concept of such robotic missions is being extended to so called on-orbitservicing. In such cases highly sophisticated robotic fixtures (arm, docking mechanism $[1,28]$ ) are used to perform servicing tasks: inspection, catching, berthing, docking, fueling, spare part replacement, etc. All these tasks require high precision and autonomy of the spacecraft while simultaneously, imposing similarly strict requirements on communication with the servicing satellite and ground operations.

In preparations for such on-orbit-servicing, several demonstration robotic missions were already performed or are now being planned [28]. For the purpose of demonstration, some of them include two spacecraft, one acting as a servicer and another one as a client. This requires a specific approach for the ground operations, especially in the case where distance between both spacecraft changes dynamically from tenths of kilometers at far range to practically zero when berthed or docked. Another case of multi-spacecraft environment is formation flight, with complexity being relevant to varying numbers of spacecraft, distances (millions of kilometers [LISA] down to hundreds of meters [TanDEM]) and aspects of communication among other factors. There are

\footnotetext{
${ }^{1}$ Ground Data System Manager, Communication and Ground Stations, DLR Oberpfaffenhofen, Wessling, Germany, marcin.gnat@dlr.de

${ }^{2}$ Ground Data System Manager, Communication and Ground Stations, DLR Oberpfaffenhofen, Wessling, Germany, peter.willburger@dlr.de
} 
additional requirements in terms of ground track accuracy, effective resolution of observation instruments, repetitiveness and payload data availability which in turn impose tight conditions for absolute and relative position, attitude, number of ground contacts and link capacity.

When discussing link capacity, we actually touch on the third area of interest. High data rate links for spaceto-ground and also within the ground segment are becoming more present than before. On the one hand it follows general development (e.g. Internet or mobile networks); on the other hand there are a lot of specific constraints which require special attention. High bandwidth is required by all the applications using video cameras (in human spaceflight and for robotic missions for inspection and telepresence) or for the data produced by the observation payload (including high resolution optical or Synthetic Aperture Radar (SAR) images and other high fidelity sensor data). The amount of data is increasing so the link capacity and quality has to increase as well, allowing full on-board memory dumps in very short contact periods. Additionally, there is an increasing number of scenarios where this high bandwidth payload information is required to be available as soon as possible, extending the need for high bandwidth over the ground network. But not only pure link bandwidth is of importance, also the questions about redundancy (is it still economically acceptable to pay two or more expensive high bandwidth links throughout the mission lifetime?), archiving and storage, monitoring of all components and finally capabilities of software and hardware are worth considering as well.

Many publications has been already written on the topic of robotics in space, stopping mostly with the robotic arm or space segment in general, whereas the communication or issues in ground segment are not usually touched upon. There are some interesting works however on signal delay in space, depending on spacecraft distance to the ground station [3]. We try to cover the next step, technically and operationally, the ground segment (Ground Station - Link - Control Center) operations with low delay, low jitter, teleoperation robotic missions with two spacecraft.

\section{Example Mission Scenarios}

Several simple communication scenarios, which we need to consider when working on the new communication concept, are presented on the figures below. These are not all the possible permutations; additionally one could expand this to cover more than two spacecraft (swarm), multiple control centers or a complicated Delay Tolerant Network (DTN) for deep space planetary mission (like Mars Rover).

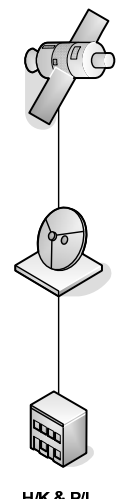

a) Simplest scenario

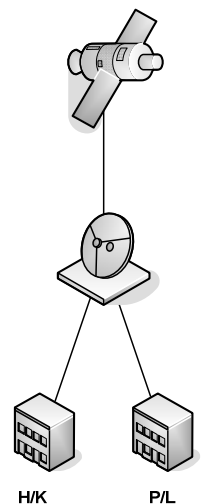

b) One spacecraft, one antenna, two control centers

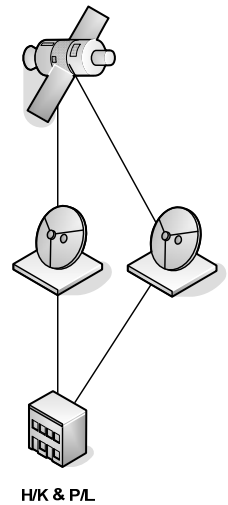

c) One spacecraft, two antennas

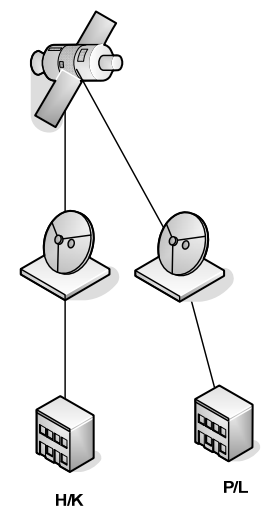

d) One spacecraft, two antennas, two control centers

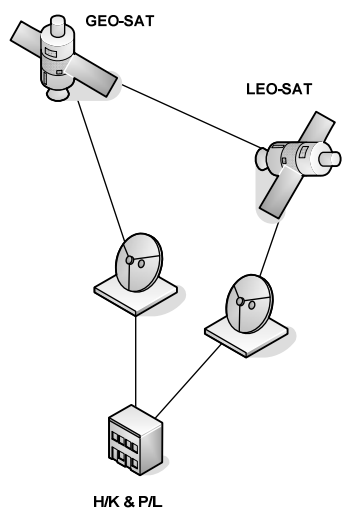

e) Spacecraft contacted directly and over GEORelay

Figure II-1 Examples of single spacecraft scenarios 


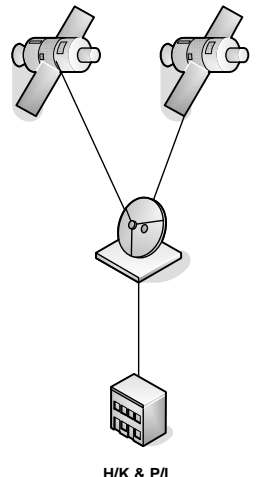

a) Dual-uplink or dual-feed antenna

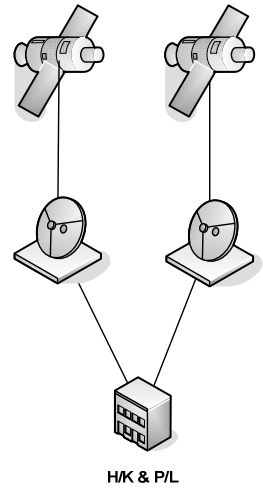

b) Two spacecrafts, two antennas

Figure II-2 Examples of two spacecraft scenarios

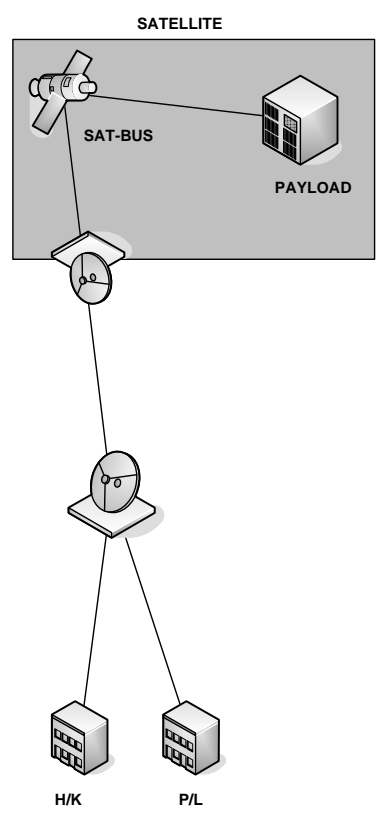

a) Payload controlled directly from second control center but over the same space link, and over the satellite bus

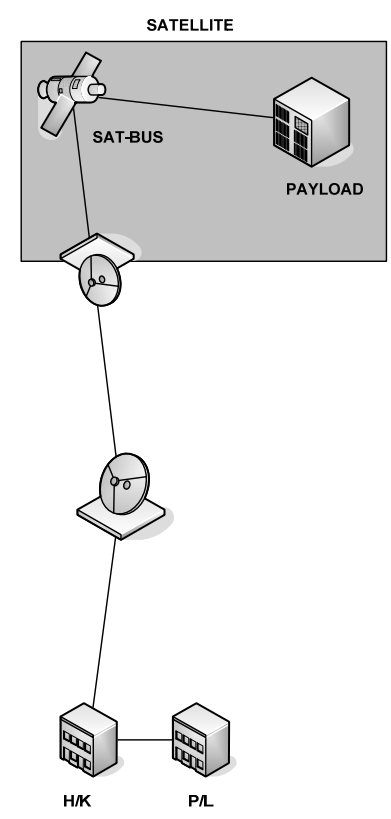

b) Payload controlled over housekeeping path

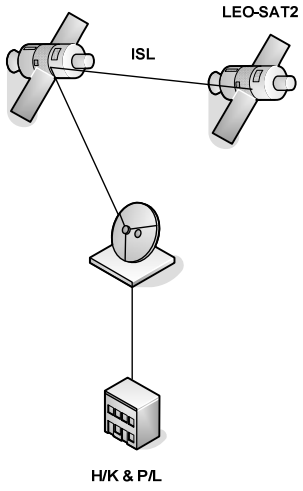

c) Two spacecrafts, housekeeping of second $\mathrm{s} / \mathrm{c}$ over ISL

\section{Figure II-3 Examples of payload control}

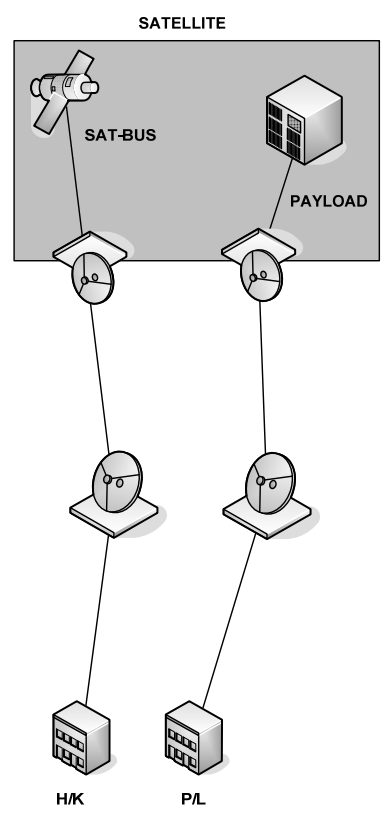

c) Payload controlled over separate ways (second control center, second antenna, second on-board transmitter)

If we would like to go more into the detail, we can divide the communication elements into a few groups, which in turn can be used to compose different permutations of the communication scenario:

- TM processing facilities (spacecraft control centers, user data center, payload control center)

- Commanding facilities (spacecraft control center, payload control center)

- Ground data connections / data links

- Ground stations and antennas (single with S or X Band, dual feed with S- and X-Band, dual uplink with two S-band uplink frequencies, etc...)

- Space links (different frequency bands like S-, X-, Ka-Band, direct link, link over GEO-Relay, Inter Satellite Link (ISL), Laser Communication)

- Spacecrafts (single, multiple, swarm, constellation, formation, client-servicer).

As soon the scenario(s) are chosen, each of these elements needs to be analyzed in terms of its capabilities and especially the interfaces between elements (i.e. ground station - data link - control center).

In the following section a few general mission profiles are presented in an example, which we have used in our analysis.

The TanDEM mission consists of two sibling satellites (TerraSAR-X and TanDEM-X) flying in a close formation (down to $200 \mathrm{~m}$ distance). The satellites have been launched separately with about two years span between. The satellites perform either Earth imaging each of its own accord, using Synthetic Aperture Radar 
(SAR) in chorus; with both SAR instruments synchronized allowing for the generation of Digital Elevation Model (DEM) image which in principle gives us a 3D map of the Earth surface [2].

The mission generates large amounts of data, which needs to be dumped in a timely manner. Additionally the formation flight requires high precision position information for both spacecraft and frequent command possibilities. These factors impose very specific requirements on the ground station network (redundant stations and connections), flight dynamics services and mission planning [2].

The PRISMA mission consists of two satellites as well. They have been started in a combined (docked) configuration, and after the commissioning phase were separated. The main satellite possesses propulsion system, whereas the secondary (or target) satellite is solely controllable with respect to the attitude (reaction wheels). The target satellite is commanded and monitored over the Inter Satellite Link (ISL) with the main satellite acting as a relay. The main satellite's payload includes cameras (for video based navigation and inspection) and GPS (for positioning and navigation). The main mission objectives are imaging, guidance, far and close navigation as well as rendezvous operations (the mission supports several other experiments; which are not relevant to this discussion). Due to the usage of the main satellite as a relay for the target, as well as for the video and other sensors, the downlink capacity is measured in the range of 1Mbps (megabits per second). Due to the scientific payload control on the ground; the scientific data archive is fed directly from the real-time data stream. This imposes a specific requirement for the ground communication (Return All Frames [RAF] service usage [11]).

The ROKVISS mission focused in principle on the payload operation, as the robotic arm was placed on the International Space Station (ISS). It was controlled over a separate space link, which was exclusively used during contact times. The space link was realized in S-Band with a 256kbps uplink data rate and $4 \mathrm{Mbps}$ downlink. On the ground, a joystick has been used with the aim to perform real-time operation of the distant (ISS) robotic arm. The feedback information for the operator on the ground was delivered as force-feedback directly back to the joystick (giving a haptic response) and video signal from the stereoscopic cameras. The main requirements could be therefore shortly summarized as high bandwidth video transmission and low delay and low jitter round trip signal propagation for the joystick-to-robotic arm operation. $[1,4,5]$

The project DEOS (DEutsche Orbital Servicing Mission) is being prepared right now. This mission is going to be a demonstrator mission, consisting of two satellites and presenting the on-orbit servicing capabilities. The spacecraft will be launched in a docked configuration. After commissioning and some experiments, the satellites will separate and the main part of the mission will begin. The main objectives are to demonstrate various relative navigation, rendezvous and docking scenarios as well as catching the client satellite (which should act as an uncooperative spacecraft) with the robotic arm, followed by the different possible servicing operations (refueling, module exchange, etc..). At the end of the mission, the controlled de-orbiting in a docked configuration will be performed. The mission presents several challenges, not only for the space segment, but also for the ground operations and infrastructure, as presented more in detail in section III.

There are also several interplanetary robotic missions, where the communication plays an important role (Mars Rovers). However these missions inherently include large signal delays and are covered with techniques like DTN (Delay Tolerant Networks) - therefore we will not deliberate more on that in our paper.

\section{Technical Solutions}

Within this section we present technical solutions and areas of possible future technical improvement for a typical telerobotics mission at DLR. The focus is put on the communication solution, whereas other elements are covered briefly.

\section{A. Low delay communication concept for robotic missions}

A new communication concept is being prepared within the generic framework for robotic missions at GSOC. In the first approach the communication concept of the ROKVISS mission [1, 4, 5] has been seen as a potentially relative simple re-use scenario. However, as the analysis of the requirements deepened, the decision to take a different approach was made. The ROKVISS-solution is still kept as a fallback possibility, nevertheless.

The main requirements which the ground communication segment needs to support can be summarized as follows:

- Uplink with 256kbps

- $\quad$ Downlink with real-time terrestrial transfer (or online) of a few Mbps

- Very low delay of the commanding loop (round-trip between sending a command and receiving feedback from the robot in space) below $100 \mathrm{~ms}$

- $\quad$ Very low jitter (few milliseconds) 
- Multiple source simultaneous commanding shall be possible (simultaneous operations of robotic payload and housekeeping)

The ROKVISS-like solution covers most of these requirements; however the question of implementation costs and general plausibility arises, especially when talking about remote ground stations. And so, the Weilheim ground station is still applicable, but for other stations there are many issues which need to be clarified (e.g. Is the modem solution applicable on distant heterogeneous networks? Is it allowed to install third party equipment at the partner site?).

The new concept is based on three derivative requirements which we defined autonomously: allow low delay and low jitter connection over larger distances, easy integration into existing infrastructure and systems and allow simultaneous commanding. These targets can be achieved (among other things) through separation of transport layer from application layer and integration with Space Link Extension (SLE) services [12].

The transport layer (and all other layers below) is to some extend dependent on the chosen technical solution for the link. Up to now typically Transmission Control Protocol (TCP) has been used. However due to the inherent mechanism for retransmission, TCP protocol is the source of jitter. This does not reject the TCP as such for the on ground communication for space applications; however in our case for the specific robotic use it seems to be interesting to look only at the User Datagram Protocol (UDP). UDP is being widely used in many streaming applications [16, 17], especially in the case where the completeness of the information is not crucial (video, voice). Now we need to consider that issue - to what extent can we allow losing some data packets, which would be the cost for lower delay and jitter? This question needs to be considered in relation to specific circumstances - on one hand UDP does not assure the delivery and sequence, but on the other hand we utilize fewer links over "open internet". Typically when ordering a link between control room and the ground station we have specific requirements to the telecom provider, including guaranteed bandwidth, availability and in general quality of service. Therefore we can assume, the delivery is guaranteed by the link (provider) itself, whereas the UDP layer guarantees us low (at least to some extent) latency and jitter.

Moreover, there are several solutions aiding the use of UDP - for example the Real-time Transport Protocol (RTP) $[14,15]$. There are also some devices on the market, which, placed on the edges of our link, take care of timely and sequenced delivery, whereas keeping jitter very low [18].

Our requirement of easy integration is going to be covered mostly by the use of SLE (or in future Cross Support Transfer Service [CSTS] and compatible protocols) at the application layer. SLE is currently widely used, and is being integrated in increasing number of agencies and other space related institutions and companies.

Up to now, with current implementations, there is no real approach for usage of SLE services for robotic mission like DEOS. The SLE as of today is more focused on safe provision of the command or telemetry, and is based on TCP/IP. Using SLE, one cannot guarantee the low jitter, although some tweaking of TCP/IP parameters (like Window Size) can improve the situation, especially in broad heterogeneous networks. Therefore one of the tasks which we are going to approach is to check the feasibility of transport-layer separated SLE protocol, or at least attempt to get some similar functionality (whereas for the future of course it would be desired to have full protocol support). In upcoming months we plan to perform experiments with SLE-like support on top of UDP.

Another topic related to the SLE is the question "Which services can be used for the robotic support?" Currently the Forward Command Link Transfer Unit (FCLTU), Return All Frames (RAF) and Return Channel Frames (RCF) services are used. Whereas the FCLTU service could be principally used (see also the issue of the multiple sources discussed in the next section) for commanding, there is potentially an issue of using the RCF (or RAF) service for robotic feedback information. This data is to be delivered to the robotic operator (or better to say the joysticks' force-feedback servo motors) in the same manner as commands - with possibly low delay and no jitter. When using RCF we have the problem of choosing the right virtual channel for the data, and also the issue of how to guarantee that each frame transported within a channel contains information we need. Additionally, all this needs to happen in constant rate (re low jitter). Also the principal data link capacity could have a possible influence on the whole performance, in case the virtual channel carries larger amounts of data. This could lead to inhomogeneous transmission and thus again end up with high jitter.

There are two possible solutions which we could cover the feedback path of the robotic data. The first one is based on the new SLE service - the Return Frame Secondary Header (RFSH). CCSDS specifies that each TM frame can contain so called secondary header section with ancillary data (up to 80 bytes) [30]. This capacity would be enough to support the force feedback information. This would guarantee the feedback information is practically carried by each single frame coming down in TM stream ${ }^{3}$. Also use of the SLE service would lead to very early separation of this information and thus asynchronous delivery over the critical path to the joystick. As

\footnotetext{
${ }^{3}$ See Acknowledgments
} 
the required data bandwidth for such information is fairly low it could be envisaged to obtain a separate physical link for the RFSH service (thus removing the risk of being degraded by other data delivered over standard RCF or RAF services on main data link).

The Figure III-1 shows the example of such configuration with CLTU's on the command path, being merged within SLE User, and as single coherent data stream forwarded over to the ground station and there radiated. On the way back, the telemetry is being split within SLE Provider, allowing all parties to receive only the telemetry they are interested in, especially allowing separate connections for sensitive data (like separate UDP link for RFSH).

The Figure III-2 presents the example of the underlying transport layer, allowing principally both housekeeping and payload operations to talk to all ground stations, choosing the link and transport layer (USP or TCP) depending on current needs.

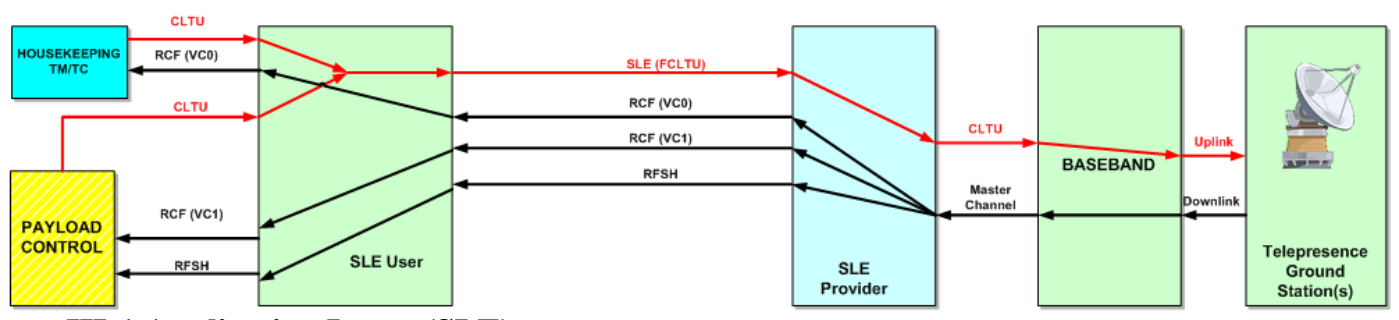

Figure III-1 Application Layer (SLE)

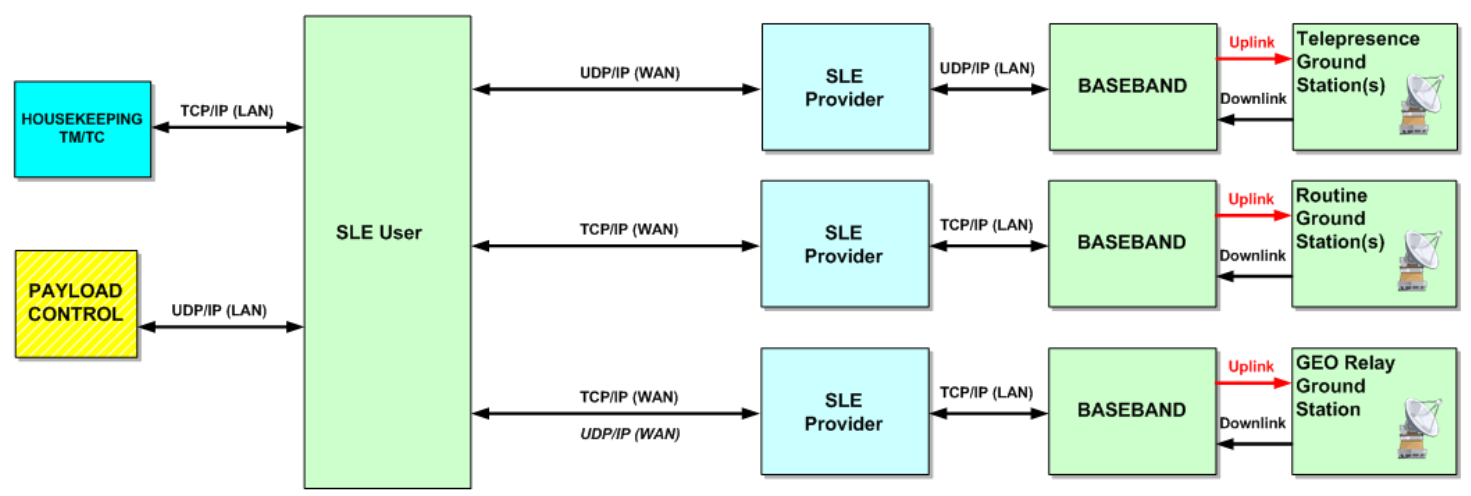

Figure III-2 Transport layer

Another possibility which we visualize for our use requires more effort (Figure III-3); however appears to be more interesting due to other aspects. This is based on the use of Space Packets [31] and its respective Forward Space Packet (FSP) [33] and Return Space Packet (RSP) services. In this case one does not even really need to define RFSH service; just a specific RSP instance could cover the robotic feedback data. The actual advantage however is given on the forward side (FSP). It allows easier multi-source commanding, where different parties may command their respective applications on board. It also closes the loop for the Communications Operations Procedure (COP-1) [32] already at the ground station, thus relieving the link to the control center. As one can see, the most software load is put on SLE Provider (merging and splitting the data), whereas the SLE User plays a role of protocol gateway.

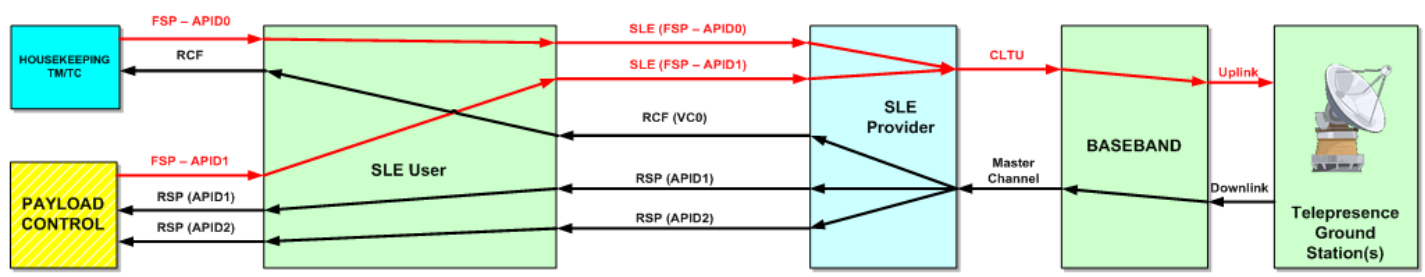

Figure III-3 Application Layer (SLE) realized with Space Packets

In case the effort for all these implementations should show to be too high at the beginning, we can imagine some specific combination of above mentioned scenarios - with RFSH for time critical robotic feedback and all other TM information provided within RCF or RAF and in the forward direction with FSP for commanding. 
Many current ground systems (TM\&TC processors, robotic control) already inherently work with space packets. Therefore we do not anticipate any paradigmatic change, whereas some basic interface implementations will need to be performed.

Finally we come to the issue of parallel commanding. As already mentioned with the use of FSP service and space packets, in principle one can rearrange the solution to the SLE service provider. This does not resolve the issue, however. We have to assume, that multiple FSP services will supply the SLE provider with asynchronous packets. The SLE service provider will need to use a complicated algorithm to sort the incoming space packets, and respectively prioritize the robotic payload commands to avoid delay while keeping the command rate constant to avoid jitter. When working on the CLTU level, one can imagine just interleaving CLTU from robotic control and common housekeeping CLTU in the manner of "every second has to be robotic" 4 . This makes the algorithm easier to implement and keep respective timings constant. Also in such case there is the possibility to decide where to perform this multiplexing (merging); at the SLE service user or at the SLE provider. The first solution makes it simpler for implementation (one is not dependent on the ground station, which needs to receive principally just one FCLTU stream). The second solution provides for asynchronous commanding over large distance possibly over a separate communication line. In such a case however a more fundamental change in the SLE service provider is needed. In both cases however the merging algorithm stays the same.

As we discussed already most of the paths between the ground station and the control center, we could still have multiple issues when dealing with the timing behavior of the equipment. We plan to perform several tests on basebands to find the optimal settings and check the principal feasibility of such. In the case where not enough performance is achieved, it is thinkable to develop a custom, integrated with the SLE provider, logical part of the baseband (Telecommand Unit [TCU] and Telemetry Unit [TMU]). These need to generate all of the information as required for the uplink, and only feed it as a bit stream into the modulation unit with Phase Change Modulation (PCM), analogue to the ROKVISS, and respectively receive a bit stream out of the modulator, decode and synchronize frames and feed it into the SLE provider for the return direction. This could constitute a high performance teleoperation station, whereas other stations with just SLE provider could principally support all the functionality, even with higher delay or not fulfilling all jitter requirements. For the operations team such a setup would give high simplification (only one configuration and teleoperations depending just on the physical link choice and the ground station). This concept offers a plug-in solution for ground segment communication.

\section{B. Dual-uplink antenna system}

In order to control multiple spacecraft flying in close formation, a possible solution which the German Aerospace Center (DLR) has implemented at its ground station in Weilheim is a dual-uplink antenna system. Since 2010 the DLR is operating TerraSAR-X and TanDEM-X in close formation with a distance of around $200 \mathrm{~m}$ between both spacecraft and an orbit of $514 \mathrm{~km}$. Until recently different antennas had to be used to control both spacecraft in parallel. Altitude, distance between both spacecraft and ground antenna diameter gives the opportunity to use one Weilheim ground antenna's 3dB-beam for transmission of telecommands, reception of telemetry and tracking of both spacecraft.

More detailed information on the operation of such antenna is presented in "Dual Operation of TerraSAR-X and TanDEM-X with one Ground Antenna" [7]. The paper shows the installation, configuration and operation of the ground antenna system as well as both spacecraft when only one ground antenna is active. An example describes results in terms of signal processing and Monitor \& Control (M\&C) system design. Operations of one and two satellites are compared and the analysis of the advantages and disadvantages will help for future works.

This solution can also be used for telerobotics missions where two independent spacecraft or in general two communication paths which fit into a ground antenna's 3dB-beam are used. Especially in the case of very close relative position, or even docked configuration, such an antenna setup can bring several advantages (saving resources, less cost and easier tracking).

Another dual use of a single dish is the dual-feed type. It is rather common type, and the principle is to use the same dish and antenna mount for two (or more) different feeds in different frequency bands (for example Sand X-Band). Whereas it is not as novel as the dual-uplink system just discussed, it can be a source of several operational issues and such a specific resource must have special attention when mission planning or just for scheduling.

\footnotetext{
${ }^{4}$ See Acknowledgments
} 


\section{Other elements of the ground system}

Other relevant elements of the ground segment include Flight Dynamics services (orbit and attitude analysis, production of orbit and attitude change commands, predictions for the ground stations, event files), Mission Operations (performing the actual monitoring and control of the spacecraft), Mission Planning, Payload Operations (control of the payload, analysis, evaluation and further processing of the payload data) and Ground Facilities or Network Operations (including Ground Data System, Scheduling, Ground Station Operations and Network Monitoring).

Due to budget constraints, the automation of ground operations is becoming a key requirement. At a minimum, routine tasks should be executed in an automatic or semi-automatic manner (using workflows or scripts). Automation brings another advantage; it allows for the monitoring and control of more spacecraft with less resources. However it still needs to be noted, that the automation comes not at zero cost, it needs to be developed, adapted to the existing system and maintained. A trade-off analysis needs to be performed, depending on the individual constraints.

Nowadays ground stations are usually remotely controlled from Network Control Centers (NCC). Routine operations are generally performed from the NCC, whereas the Launch and Early Orbit Phases (LEOP's) might be supported directly from the ground station. This extends the need for complete monitoring \& control and fault reaction, but also routine remote actions performed from a network control center.

All this requires a large effort on the part of software development for automation, monitoring and control. Such systems need to be scalable, redundant, and reliable and shall be fully integrated into the existing environment. Another aspect in terms of cross support is the availability of a standardized interface for monitoring information as a minimum.

The scheduling system is one of the central elements of the ground system. The complexity ranges from very simple implementations to very sophisticated ones. In principle, however, this is an expanding area, and its several aspects shall also be covered by the Consultative Committee for Space Data Systems (CCSDS) in the near future [13].

Scheduling needs a good interface with the Mission Planning System (MPS) to exchange all the information required to plan the specific contacts with the spacecraft. The information flow is needed not only in one direction (a schedule request based on general project constraints) but also in the form of feedback to the project or even explicitly to the mission planning system. Already scheduled contacts are used to plan further actions with the project (such as offline data transfers or the triggering of data processing). Also the information about the current status of the ground stations is transferred allowing for better long term planning (i.e. information about antenna maintenance).

When talking about the interface to the MPS, we touch on the main issue in today's scheduling systems different data formats. There is some effort (as from the CCSDS) to standardize the interface and file format for the scheduling information. Currently, however, the scheduling system needs to support a number of different formats and ways of exchanging the information. Much schedule information is exchanged, for example, in simple text form via e-mail. This is rather error prone, and hard to verify. The standardized and primarily automated processing systems should be pursued, leaving however special tasks (like ad-hoc scheduling, emergency and conflict resolution) in the hands of a human (scheduling officer).

Specific examples of scheduling issues which can appear during parallel support became obvious during the TanDEM mission some time ago. The mission is performed by two satellites flying in close formation [2], operating the same type of instrument and contacting the Earth in the same frequency bands (S-Band for housekeeping and X-Band for payload). Due to the close distance of the satellites it was discovered, that it is possible to perform parallel contact with only one antenna equipped with a dual-feed [7]. As long as the contact requests for the two satellites are consolidated there is no issue there. However this is usually the case only for housekeeping data. The payload data contacts are being planned much more dynamically and the requests are often received from different sources. As it happened; a housekeeping contact was already planned in S-Band with one of the satellites in close formation, when another request for a data dump in X-band on the same antenna but the other spacecraft for was received. Unfortunately, at that moment, no one was aware of this specific situation. The X-Band request arrived significantly later and the scheduling software was not prepared. The outcome was that the first request (S-Band housekeeping) was deleted and overwritten by the X-Band request. Currently such constellations of requestor-antenna-spacecraft have been implemented into procedures and we are working on changes in the scheduling software to support such cases. 


\section{Challenges for Operations}

In this section we have collected several issues or challenges which we consider important for close formation flight and telerobotics missions. It does not cover all aspects of ground operations; we focus solely on ground communication and infrastructure.

During formation flight missions, one of the issues is the Radio Frequency Interference (RFI) between the satellites, or one satellite influencing the ground contact of the another one. It is possible for two spacecraft to have the same or very similar space link frequencies (which is especially true in X-Band and for payload data, as they typically use almost the whole available bandwidth). The results are massive data drops or even complete lost passes. It is crucial to perform coordination in the early phase (International Telecommunication Union [ITU] [21] or Space Frequency Coordination Group [SFCG] [20]), to account for RFI's and respectively perform planning to avoid interferences. Also later, shortly before mission begin and during the mission this coordination must be continued. The proper localization (and their assignment to the spacecraft) of the ground stations can help avoiding these issues.

Crucial for any mission, but especially for the robotics and close formation is the Flight Dynamics support and products. High availability of these services and products is one of the main tasks of the Ground Data System. This can be approached from different sides, and so the frequent availability of tracking data helps increment the orbit estimation precision. Timely distribution of visibility and orbit information to all interested parties (mission, ground stations) helps the better planning of activities. Often in case of critical maneuvers the immediate availability of specific attitude or orbit information becomes a major element of the mission operations. It is important to support the dissemination of this data with controlled data transfers, backups and frequent tests of data distribution paths.

Missions with multiple spacecraft and very specific payloads which need to be controlled directly by its own engineering team, compose a very specific environment for scheduling services. What was previously not an easy task becomes even more complicated, and one has to ask the question "How far can this scheduling be handled strictly by the operator?" For simple missions (like case a on the Figure II-1 Examples of single spacecraft scenarios) the scheduling process is based on the "first in, first out" principle, and was serialized. Mostly inter-mission conflicts and some priority regulations are the cause of intense work. With the missions which we are discussing here, there are additional complications; the booking of the same antenna resource for two spacecraft (dual-uplink or dual-feed antennas), parallel requests from two different sources, etc. As long as the number of missions and cross supports remains lower than just a few per day, with some effort and training it can be handled with the old tools. When this frequency increases however, the support of a more sophisticated scheduling system is required. Such a system could be realized on the platform of web services for example, allowing easy user access and extensibility.

The mission operations team is also one of the topics which require closer regard. Particularly the cost of the personnel in long lasting missions becomes a main cost driver. For close formation and robotics mission the team needs to be expanded for crucial operations (maneuvers, teleoperation, etc.). Afterwards there is often a need for observation of the spacecraft for a longer period of time - which easily leads to 24/7 operations and a full three shift setup. Thus there is a strong push for automation of specific routine operations, more requirements to the Failure Detection, Isolation and Recovery (FDIR) mechanisms on-board and better monitoring of all elements (with, for example, direct notification of on-call personnel in case of problems).

When planning robotic mission, one has to analyze the availability of contacts to the spacecraft. Typically the spacecraft in LEO has a maximum of ca. 10 minutes visibility with a ground station. Assuming a small buffer for locking on to the spacecraft and synchronizing, what is left for real operation is something in the range of 6 to 8 minutes. It is possible to plan contacts such a way, that one becomes virtually one long contact period (composed out of consecutive single contacts). This however causes operational issues, as the handovers need to be planned carefully and there will be still gaps in commanding. Such contacts in a series can't be however planned for every orbit, as the ground stations are not geographically evenly placed. Also issues with respect to ground link (availability of equal quality link to all stations) can arise. As a solution, a link over a GEO-Relay satellite is proposed. This gives homogenous contact times to the robotic spacecraft in ranges of 30 to 45 minutes. However this comes with the cost of longer space signal flight time (for a typical "ground to GEO to ground" stretch, one must calculate a ca. 500ms round trip time). This makes real-time operation with joystick feedback more difficult and puts very strict requirements on the terrestrial link (which then needs to compensate for the extra time used via the GEO-Relay). Example of such experiments is presented in [6].

Ground communication lines become one of the most important parts of the ground segment for real-time control of telerobotics missions. The capacity of the link needs to be high enough to avoid backlog, and allow low delay commanding and feedback, simultaneously with one more real-time video signals. Where for normal housekeeping and routine operations of previous missions one needed a solution in the range of 64kbps (ISDN), suddenly for such a robotic mission the link capacity grows to a minimum of a few megabytes per second. This drives the cost, particularly if we plan to use multiple ground stations as discussed above. Also some technical 
issues can arise, when crossing national borders we are probably confronted with a change of telecom provider and inherently the technology it uses. This can again end up with longer delay times or sudden jitter. Therefore extensive testing of ground communication, before the actual mission start, is required. Due to several complicated elements in the system (multiple receivers, several commanding sources); end-to-end testing is strongly suggested.

One additional item regarding signal delay should also be noted. A slowly increasing signal delay stays unnoticed for a relatively long time, causing sudden interruption either in communication protocols (SLE) or within TM\&TC processors. Unpredictable data drops will result. Therefore, from the operations team sort of current delay monitoring should be requested. If it is displayed in real-time or otherwise communicated to the team, it is of a second order importance. Essential is that the operations team can timely prepare for worst condition, altering their plan, changing experiments or even completely postponing an action, until line conditions improve.

As an example for the above mentioned areas, unexpected problems occurred when using Virtual Private Network (VPN) connections over Internet with data rates of 1Mbps [8]. Something what does not look challenging for most of the home Internet users, showed up as hard work to get it running. The mission required $1 \mathrm{Mbps}$ real-time downlink from the spacecraft directly to the TM/TC processing software. As it turned out, the connection over the internet was unreliable and the actual bandwidth considerably below the requested $1 \mathrm{Mbps}$. This led to serious data drops and thus to problems feeding the archive with scientific data. The solution was to implement the SLE service in the on-line complete mode (this has at least guaranteed that there are no data drops), however -it has had the result that the total delay of the "real-time" data have to be measured in minutes; the backlog is considerable. In the final stage however, a separate connection (over the Multi-Protocol Label Switching [MPLS] network [19]) has been implemented. This was an important lesson: connection lines are cost drivers, and due to the availability and "daily applicability" of the Internet, there is imaginable temptation to use it as an alternative. One can do that, but there is a need to perform enough tests in advance and also be prepared, that in some cases the VPN over Internet won't be a viable solution at all.

\section{Conclusion \& Outlook}

In our paper we have tried to collect different topics regarding close formation flight and general concerns with multiple spacecraft, high data rate connections and real-time applications, showing the analysis which one needs to perform in a preparation for such missions. Special focus was put on an early concept of the communication setup for future robotics, multiple satellite and multiple control entity missions.

First plausibility tests for new communication concept and the work on prototypes are started and within a year the base results are expected, which will be followed by step by step implementation. The ultimate target is to create a communication system, which will allow flexible and easy usage in a cross support environment (inter-agency support) and will fulfill all the payload requirements.

\section{Appendix A \\ Acronym List}

$\begin{array}{ll}\text { CCSDS } & \text { Consultative Committee for Space Data Systems } \\ \text { CSTS } & \text { Cross Support Transfer Services } \\ \text { COP-1 } & \text { Communications Operation Procedure } \\ \text { DEOS } & \text { DEutsche Orbital Servicing Mission } \\ \text { DTN } & \text { Delay Tolerant Network } \\ \text { FCLTU } & \text { Forward Command Link Transfer Unit } \\ \text { FD } & \text { Flight Dynamics } \\ \text { FDIR } & \text { Failure Detection, Isolation and Recovery } \\ \text { FSP } & \text { Forward Space Packet } \\ \text { GEO } & \text { Geostationary Earth Orbit } \\ \text { GSOC } & \text { German Space Operations Center } \\ \text { H/K } & \text { Housekeeping }\end{array}$




$\begin{array}{ll}\text { IP } & \text { Internet Protocol } \\ \text { ISL } & \text { Inter Satellite Link } \\ \text { ISDN } & \text { Integrated Services Digital Network } \\ \text { ITU } & \text { International Telecommunication Union } \\ \text { LEO } & \text { Low Earth Orbit } \\ \text { LEOP } & \text { Launch and Early Orbit Phase } \\ \text { MPLS } & \text { Multi-Packet Level Switching } \\ \text { MPS } & \text { Mission Planning System } \\ \text { NCC } & \text { Network Control Center } \\ \text { OOS } & \text { On-Orbit Servicing } \\ \text { P/L } & \text { Payload } \\ \text { RAF } & \text { Return All Frames } \\ \text { RCF } & \text { Return Channel Frames } \\ \text { RFI } & \text { Radio Frequency Interference } \\ \text { RFSH } & \text { Return Frame Secondary Header } \\ \text { RTP } & \text { Real-time Transport Protocol } \\ \text { SLE } & \text { Space Link Extension } \\ \text { SFCG } & \text { Space Frequency Coordination Group } \\ \text { TC } & \text { Telecommand } \\ \text { TCP } & \text { Transmission Control Protocol } \\ \text { TM } & \text { Telemetry } \\ \text { UDP } & \text { User Datagram Protocol } \\ \text { VPN } & \text { Virtual Private Network }\end{array}$

\section{Appendix B \\ Glossary}

Antenna feed

Baseband

Force-feedback

Housekeeping

Jitter

Round-Trip Time
An antenna feed refers to the components of an antenna which feed the radio waves to the rest of the antenna structure, or in receiving antennas collect the incoming radio waves, convert them to electric currents and transmit them to the receiver.

Baseband is an adjective that describes signals and systems whose range of frequencies is measured from close to 0 hertz to a cut-off frequency. In space communication the baseband frequency is also the name for intermediate frequency between the modulator and the up-converter (and typically equals $70 \mathrm{MHz}$ ). Colloquially the device containing modulator and as well as telecommand and telemetry units is called baseband unit or just baseband.

or haptics, is a tactile feedback technology which takes advantage of the sense of touch by applying forces, vibrations, or motions to the user.

Colloquial name for the operations or data (telecommands and telemetry) which serve the base operation of the satellite bus (power, thermal, attitude, etc...).

Jitter is the undesired deviation from true periodicity of an assumed periodic signal.

The round-trip delay time (RTD) or round-trip time (RTT) is the length of time it takes for a signal to be sent plus the length of time it takes for an acknowledgment of that signal to be received. More specific in the case of teleoperations, this is the time from the command being sent (moved 
joystick) to the moment when the force-feedback information is being received.

S-Band

Teleoperation

Telepresence

Telerobotics

X-Band
Frequency range of 2-4 GHz (IEEE)

Means "doing work at a distance", whereas in our specific case we just refer to the distant robotic arm operations performed remote with joystick.

Means "feeling like you are somewhere else", whereas in our case we refer to that term as to the situation when we can observe (via video and sensory information) the distant spacecraft and monitor in real-time its robotic operations.

The area of robotics concerned with the control of robots from a distance.

Frequency range of 8-12 GHz (IEEE)

\section{Acknowledgments}

Authors would like to acknowledge Stefan Funk (Astrium GmbH) with his ideas of interleaving CLTU's and usage of the frame secondary header for feedback data on the Space Link.

\section{References}

${ }^{1}$ K. Landzettel, A. Albu-Schäffer, C. Preusche, D. Reintsema, B. Rebele, G. Hirzinger, "Robotic On-Orbit Servicing DLR's Experience and Perspective" - Proceedings of the 2006 IEEE/RSJ International Conference on Intelligent Robots and Systems, October 9-15, 2006, Beijing, China

${ }^{2}$ H. Hofmann, R. Kahle, "The TanDEM-X Mission Operations Segment: Close formation flight: Preparation and First Experiences". - SpaceOps 2010

${ }^{3}$ E. Stoll, J. Letschnik, U. Walter, C. Preusche, G. Hirzinger, "Concept of an Algorithm to Determine the Signal Delay Time for Telepresence Space Applications“

${ }^{4}$ K. Landzettel, A. Albu-Schäffer, B. Brunner, A. Beyer, R. Gruber, E. Krämer, C. Preusche, D. Reintsema, J. Schott, B.-M. Steinmetz, H.-J. Sedlmayr, G. Hirzinger, "ROKVISS, Verification of Advanced Light Weight Robotic Joints and Tele-Presence Concepts for Future Space Missions"

${ }^{5}$ C. Preusche, D. Reintsema, K. Landzettel, G. Hirzinger, "Robotics Component Verification - ISS ROKVISS Preliminary Results for Telepresence“- Proceedings of the 2006 IEEE/RSJ International Conference on Intelligent Robots and Systems, October 9-15, 2006, Beijing, China

${ }^{6}$ E. Stoll, J. Letschnik, U. Walter, J. Artigas, P. Kremer, C. Preusche, G. Hirzinger, "On-Orbit Servicing, Exploration and Manipulation Capabilities of Robots in Space", IEEE Robotics \& Automation Magazine, December 2009

${ }^{7}$ D. Dikanskis, K. Wiedemann, M. Preuß, "Dual Operation of TerraSAR-X and TanDEM-X with one Ground Antenna", AIAA SpaceOps 2012 Conference, June 11-15, Stockholm, Sweden

${ }^{8}$ C. Furtuna, W. Kruse, C. Garcia, "SLE experience over unreliable data links", SpaceOps 2012, June 11-15, Stockholm, Sweden

${ }^{9}$ F. Sellmaier, F., Boge, T., Spurmann, J., Gully, S., Rupp, T., Huber, F., “On-Orbit Servicing Missions: Challenges and solutions for Spacecraft Operations", AIAA SpaceOps 2010 Conference, Huntsville, Alabama, USA

${ }^{10}$ S. Eberle, A. Ohndorf, R. Faller, "On-Orbit Servicing Mission Operations at GSOC “, AIAA SpaceOps 2010 Conference, Huntsville, Alabama, USA

${ }^{11}$ CCSDS 911.1-B-3, Space Link Extension - Return All Frames Service Specification, Blue Book, Issue 3, January 2010

${ }^{12}$ CCSDS 910.4-B-2, Cross Support Reference Model - Part 1: Space Link Extension Services, Blue Book, Issue 2, October 2005

${ }^{13}$ CCSDS 910.11-B-1, Space Communication Cross Support - Service Management Service Specification, Blue Book, Issue 1, August 2009

${ }_{15}^{14} \mathrm{http} / / /$ en.wikipedia.org/wiki/Real-time_Transport_Protocol

$15 \mathrm{http} / / /$ tools.ietf.org/html/rfc3550

${ }^{16} \mathrm{http}: / /$ tools.ietf.org/html/rfc768

17 http://en.wikipedia.org/wiki/User Datagram Protocol

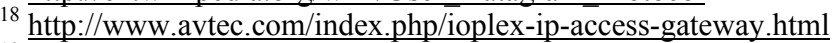

19 http://en.wikipedia.org/wiki/MPLS

20 https://www.sfcgonline.org/

${ }^{21}$ http://www.itu.int/

22 "UN Space Debris Mitigation Guidelines", UN Office for Outer Space Affairs, 2010. 
23 E. A. Taylor and J. R. Davey, "Implementation of debris mitigation using International Organization for Standardization (ISO) standards", Proceedings of the Institution of Mechanical Engineers: G, Volume 221 Number 8 (1 June 2007)

${ }^{24}$ J. W. Campbell, Colonel, USAER, "Using Lasers in Space: Laser Orbital Debris Removal and Asteroid Deflection”, December 2000, Occasional Paper No. 20 Center for Strategy and Technology Air War College

${ }^{25}$ M. H. Kaplan, "Space Debris Realities and Removal", Improving Space Operations Workshop Spacecraft Collision Avoidance and Co-location, 25 May 2010

${ }^{26}$ C. Kaiser, E. Bellido, P. Hofmann, "Space Debris Mitigation Using On-Orbit Servicing Solutions”, IAC 2010

${ }^{27}$ K.Landzettel, B.Brunner, K.Deutrich, G.Hirzinger, G.Schreiber, B.-M. Steinmetz, "DLR's Experiments on the ETS VII Space Robot Mission.” In Proc. of the 9th International Conference on Advanced Robotics (ICAR), Tokyo, Japan

${ }^{28}$ D. Reintsema, K. Landzettel, G. Hirzinger, "DLR's Advanced Telerobotic Concepts and Experiments for On-Orbit Servicing", DLR

${ }^{29}$ K. Yoshida, "Achievements in Space Robotics, Expanding the Horizons of Service and Exploration", IEEE Robotics \& Automation Magazine, December 2009

${ }^{30}$ CCSDS 132.0-B-1, TM Space Data Link Protocol . Blue Book. Issue 1. September 2003

${ }^{31}$ CCSDS 133.0-B-1, Space Packet Protocol. Blue Book. Issue 1. September 2003

${ }^{32}$ CCSDS 232.1-B-2, Communications Operation Procedure-1, Blue Book. Issue 2. September 2010

${ }^{33}$ CCSDS 912.3-B-2, Space Link Extension - Forward Space Packet Service Specification, Blue Book, Issue 2. July 2010 